\title{
I Vergangene Versprechen der Ersten Republik: Der Zusammenhang sozialer und gleicher Rechte von Minderheiten am Beispiel Kärnten/ Koroška
}

\author{
Maria Clar
}

\section{Zusammenfassung}

Nach dem Ersten Weltkrieg zerfielen in Europa Staaten und es stellten sich Fragen der Grenzziehung - so auch in Kärnten/Koroška. Im Oktober 1920 wurde im mehrheitlich slowenischsprachigen Teil über die staatliche Zugehörigkeit abgestimmt. Ausschlaggebend für den Ausgang waren das politische System der Ersten Republik und die damit verbundenen Versprechen sozialer und gleicher Rechte sowie wirtschaftliche Faktoren. Hundert Jahre später zeigt sich, dass nicht alle Versprechen gehalten wurden, und es regt zu Überlegungen an, wie Rechte von Minderheiten in Demokratien eingebunden werden können. Im vorliegenden Beitrag wird, mit Fokus auf die Protokolle der provisorischen Kärntner Landesversammlung und des Kärntner Landesrates, beleuchtet, wie Mitbestimmung, soziale Rechte sowie das Verhältnis von Minderheit(en) und Mehrheit(en) Eingang in eine folgenreiche politische Entscheidung gefunden haben. Dabei wird sichtbar, wie soziale Fragen mit Möglichkeiten des Spracherhalts zusammenhängen. Daran anschließend kann die Abhängigkeit von Minderheitenrechten von der Umsetzungsbereitschaft hegemonialer Gruppen diskutiert werden.

Schlagwörter: Sozialpolitik, Gleichberechtigung, Minderheiten, Mitbestimmung, Kärnten

Broken promises of the First Republic:

The connection between social and equal rights of minorities in Carinthia, Austria

Abstract

After the First World War, states disintegrated in Europe, and in some regions, questions arose about the demarcation of borders. This was also the case in the then mainly Slovenian-speaking part of Carinthia in southern Austria. In October 1920, a vote was taken on state affiliation. The decisive factor was the political system of the so-named First Republic and the associated promises of social and equal rights as well as economic factors. A hundred years later, it becomes clear, that not all promises have been kept. It encourages reflection on how the rights of minorities can be incorporated into democracies. The present article, with a focus on the minutes of the provisional Carinthian federal-state assembly and the Carinthian provincial government, examines how participation, social rights, and the relationship between minority and majority, have found their way into a momentous political decision. It becomes clear how social issues and the possibility of language retention are an intertwined part of the regional history of Carinthia. Subsequently, the dependence of minority rights on the willingness of hegemonic groups to implement them can be discussed.

Keywords: social policy, equality, minorities, participation, Carinthia 


\section{Einleitung}

Nachdem sowohl das Kraljevstvo Srba, Hrvata i Slovenaca/Königreich der Serben, Kroaten und Slowenen (SHS) als auch die Republik Österreich nach dem Ersten Weltkrieg Gebietsforderungen bezüglich des Gebietes Südkärnten/Južna (Avstrijska) Koroška und Teile der Steiermark/Štajerska stellten, kam es in Kärnten/Koroška zu Kämpfen zwischen SHS-Truppen und „Kärntner Freiwilligenverbänden“, dem sogenannten „Kärntner Abwehrkampf“. Daraufhin wurde bei der Pariser Friedenskonferenz ein Plebiszit in den großteils slowenischsprachigen Regionen Kärntens/Koroškas zur Frage der Grenzziehung beschlossen. Nach einem Propagandawahlkampf stimmten am 10. Oktober 1920 in der vom SHS-Staat verwalteten Zone A ${ }^{1}$ 59,04 Prozent (22.025 Abstimmungsberechtigte) für Österreich und 40,96 Prozent (15.278 Personen) für den SHS-Staat. Über 10.000 Slowenischsprachige, also ca. jede zweite Stimme, votierten dabei für Österreich. Ein wichtiges Argument waren die unterschiedlichen politischen Systeme - Österreichs neu gegründete Republik auf der einen Seite und der SHS-Staat als Monarchie auf der anderen Seite. Dies war vor allem auch eine soziale Frage, denn eine Republik mit gleich zu Beginn verkündeten Erweiterungen von sozialen Rechten ist für kapitalärmere Schichten, die in den Abstimmungszonen mehrheitlich slowenischsprachig waren, ansprechender als eine Monarchie. Ein weiterer Antrieb war, dass der slowenischen Sprache die Gleichstellung zugesichert wurde - beispielsweise von der provisorischen Kärntner Landesversammlung im Vorfeld der Volksabstimmung. Dazu kam, dass die wirtschaftlich und beruflich wichtigen Städte Villach/Beljak und Klagenfurt/Celovec jenseits der neuen Staatsgrenze liegen würden (siehe u. a. Obid et al. 2002; Malle/Entner 2003; Holzer 2008; Ogris 2010; Valentin 2013).

Nach dem Ende der Monarchie stellte die Ausrufung der Republik Österreich eine politische Umwälzung auf allen Ebenen dar. Auch unter dem Druck der Russischen Revolution wurden Sozialgesetze verabschiedet, die vor allem Lohnabhängigen Rechte

1 Die strittigen Gebiete wurden in zwei Zonen geteilt: A und B. Zone A wurde bis zur Abstimmung vom SHS verwaltet und war mehrheitlich slowenischsprachig. In der von Österreich verwalteten Zone B, in welcher auch Klagenfurt/ Celovec lag, würde es nach einer Entscheidung der Zone A für den SHS-Staat zur Abstimmung kommen und bei einer Entscheidung für Österreich in der Zone A würde es zu gar keiner Abstimmung in der Zone B kommen. zusprachen, die sie bis dahin so noch nicht hatten (Malina/Renner 2008: 25-26). Der vorliegende Beitrag fokussiert sich auf das Bundesland Kärnten/Koroška² und die Zeit von November 1919 bis Frühjahr 1921. Es wird auf sozialpolitische Fragestellungen Bedacht genommen und andere für den Ausgang des Plebiszits relevante Aspekte, wie internationale politische Entwicklungen oder der Propagandawahlkampf, werden außen vorgelassen. Bundespolitische Entscheidungen und Diskussionen auf Ebene einzelner Gemeinden sind auch nicht Teil dieser Betrachtung.

Der Artikel wird mit der These eingeleitet, das Sprachgebrauch und -erhalt sowie Möglichkeiten der Mit- und Selbstbestimmung eine Statusfrage sind. In diesem Zusammenhang wird auch auf die Rolle der Sozialdemokratischen Partei in Kärnten/Koroška hingewiesen, der drei Versuche einer Gleichstellung zuzusprechen sind. Darauf aufbauend wird auf den Zusammenhang von Minderheitenzugehörigkeit und Klasse näher eingegangen. Dabei kann über einen Zeitsprung vom 19. Jahrhundert bis in die frühen 2oooer einerseits gezeigt werden, dass ökonomische Abhängigkeit sprachliche Assimilation fördert, und andererseits, dass das Sprechen einer dominierenden Standardsprache mit der Hoffnung auf sozialen Aufstieg verbunden wird. Anhand des historischen Kontextes, vor allem auf Basis von Protokollen der provisorischen Kärntner Landesversammlung ${ }^{3}$ und des Kärntner Landesrates ${ }^{4}$ von November 1919 bis Frühjahr 1921, die im Kärntner Landesarchiv verwahrt sind, wird auf die Versprechen sozialer und gleicher (Sprach-) Rechte und deren Nicht-Erfüllung im Rahmen der Ersten Republik fokussiert. Dabei wird deutlich, dass vor dem Plebiszit Versprechen gemacht wurden, die direkt auf die kapital-ärmeren und slowenischsprachigen Stimmberechtigten abzielten. Durch den Vergleich mit den Protokollen direkt nach der Abstimmung kann gezeigt werden, dass es leere Versprechen waren. Bei den sozialpolitischen Fragen kann dies zum Teil an der Unsicherheit bezüglich der Zuständigkeiten liegen. Bei der sprachlichen Gleichstellung, wie die Geschichte weiter zeigt, ist es allerdings vor allem dem Deutschnationalismus zuzuschreiben. Der historische Blick auf

2 Steiermark/Štajerska hat ebenfalls eine ähnliche zweisprachige Geschichte mit Slowenisch und Deutsch - da es vor Ort allerdings keinen Plebiszit gab und die Regionalgeschichten zum Teil abweichen, ist sie nicht Teil dieser Auseinandersetzung.

3 Heute der Landtag.

$4 \quad$ Heute die Landesregierung. 
die Geschehnisse vor 100 Jahren ermöglicht es, Kontinuitäten nachzuzeichnen sowie das Zusammendenken von ökonomischen Existenzfragen, Spracherhalt und Machtpolitik zu diskutieren. Da in demokratischen Prozessen Minderheitenrechte von Mehrheitsmeinungen abhängig sind, stellt dies das abschließende Kapitel dieses Artikels dar. Anhand dieser Auseinandersetzung soll einerseits gezeigt werden, wie verwoben Möglichkeiten des Spracherhalts mit sozialpolitischen Fragestellungen sind, und andererseits das Verhältnis von Mehrheit und Minderheit kritisch hinterfragt werden.

\section{Spracherhalt und Mitbestimmung als Statusfrage}

Dass ökonomische Abhängigkeiten mit Fragen des Sprachgebrauchs eng verknüpft sind, soll vor allem in den nächsten beiden Kapiteln dargestellt werden. Dabei geht es um die Verknüpfung der wirtschaftlichen Existenz mit der Verwendung der vorherrschenden Sprache. Auch welche Modelle der Mitbestimmung überhaupt angedacht werden können, scheint oft eine Statusfrage zu sein. Dabei zeigen bekannte Beispiele, dass es sowohl um Besser- als auch um Schlechterstellung geht. So gehört Südtirol zu den reichsten Regionen Italiens und dient zeitgleich als Paradebeispiel für die autonome Verwaltung einer Minderheit in einem Nationalstaat mit anderer Mehrheitssprache. Auch Katalonien ist eine der reichsten Regionen Spaniens, wobei Spanien - gerade im Widerspruch zu Frankreich - ein besonders weitreichendes regionales Autonomiesystem hat. Am anderen Ende wirtschaftlicher, finanzieller und sozialer Möglichkeiten stehen in vielen Ländern Rom:nja und Sinti:zze. Aufgrund ihrer marginalisierten Position wird bei diesen Gruppen seltener bis gar nicht an Varianten der Selbst- oder Mitbestimmung gedacht.

Dabei geht es nicht ausschließlich um die Statusfrage der Sprecher:innen, sondern auch um die Wertung der Sprache selbst. Nach Pierre Bourdieu gibt es einen ,sprachlichen Markt', welcher normiert und standardisiert ist und in welchem alle möglichen Formen von Sprache mit jener Sprache verglichen werden, die in diesem speziellen Kontext als legitim erachtet wird. Dies ist beispielsweise die herrschende Sprache innerhalb nationalstaatlicher Grenzsetzungen, welche sich gegen andere ,Sprachpraxen' durchsetzt - sowohl gegen andere Sprachen wie gegen andere, sozial konnotierte ,Sprachgebräuche‘. Sprache an sich wird dabei oft mit „der offiziellen Sprache einer politischen Einheit" gleichgesetzt (Bourdieu 2005: 49-50).

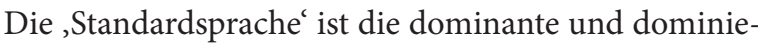
rende. Die Kenntnis dieser Sprache wird mit Vorteilen und sozialem Aufstieg verbunden. Deshalb wird sie ständig produziert und reproduziert. Andere Sprachen oder Sprachformen werden mittels Strategien wie Trivialisierung oder Marginalisierung der dominierenden Sprache untergeordnet (Wohlfahrter 2014: 42-43). So auch in Kärnten/Koroška, wo versucht wird, den Gebrauch der slowenischen Sprache über Begriffe wie ,Windisch", als Zeichen dafür, dass es kein Slowenisch ist, was gesprochen wird, zu trivialisieren und abzuwerten (Clar 2016: 14).

So hängt auch das vermehrte Interesse an Zweiund Mehrsprachigkeit in Kärnten/Koroška der vergangenen Jahre mit dem Entstehen und der Erweiterung der Europäischen Union zusammen. Nach dem Zusammenbruch Jugoslawiens wurde Slowenisch Staatssprache und mit dem Beitritt Sloweniens zur EU 2004 europäische Amtssprache (Busch 2008: 2). Dies stellt nicht nur eine Aufwertung der Sprache Slowenisch dar, sondern zeigt auch, dass Prestige auf unterschiedlichen Ebenen ein wichtiger Aspekt für den Erhalt von Sprachen ist. Wie dieser Beitrag versucht nachzuzeichnen, führt wirtschaftlicher Druck zu Assimilation. Zeitgleich waren soziale Fragen ausschlaggebend für den Ausgang des Plebiszits in Kärnten/Koroška, der im historischen Verlauf zu weiterer Assimilierung geführt hat.

Abgesehen von der Zeit, als nach dem Zweiten Weltkrieg die Kärntner Politik unter dem Druck und der Beobachtung der Alliierten stand, gab es in den vergangenen 100 Jahren drei Mal Versuche, eine minderheitenfreundliche oder gleichberechtigende Politik in Kärnten/Koroška zu leben. 1924 trat die Sozialdemokratische Partei aus dem Kärntner Heimatdienst aus. Der Kärntner Heimatdienst war eine im Vorfeld des Plebiszits von allen Parteien der provisorischen Landesversammlung (in der kein:e Vertreter:innen der slowenischsprachigen Kärntner:innen Mitglied waren) eingerichtete Koordinationsstelle, die für den Wahlkampf und die Propaganda, das mehrheitlich deutschsprachige Österreich zu wählen, zuständig war. Zeitgleich riet dieselbe Partei ihren Mitgliedern auf-

5 ,Windisch ' wurde in den 1920er-Jahren als politischer Begriff verwendet, der die slowenischsprachige Bevölkerung in ,slowenisch' und ,heimatfeindlich' sowie ,windisch und ,heimatfreundlich' aufteilen sollte mit dem Ziel, die Sprachgruppe zu schwächen und letztendlich zu delegitimieren. Auf den politischen Hintergrund des Begriffes wird im historischen Kontext noch näher eingegangen. QUARTERLY IIIIIIIIIIIIIIIIII

37 
grund des deutschnationalen Charakters der 10.-Oktober-Feiern, die jährlich an den Ausgang des Plebiszits erinnern sollen, davon ab, an diesen teilzunehmen. Ein Jahr später, 1925, initiierten die Sozialdemokrat:innen eine Kommission, in der alle Parteien und zwei Vertreter der Kärntner Slowen:innen über die ,Minderheitenschulfrage verhandelten. 1927 kann dieser Versuch als gescheitert bezeichnet werden, nachdem die meisten deutschsprachigen Parteien für Zusagen Einträge aller sich zur slowenischen Sprache Bekennender in ein Kataster verlangten und zeitgleich keine Zugeständnisse bei einer Verwaltungsübertragung beim utraquistischen Schulwesen machten - mit der Begründung, dass sie die ,Windischen' vertreten und diese würden in keine slowenischen Schulen gehen wollen. Damit zog sich auch die sozialdemokratische Partei wieder aus der Position zurück, sich für die Gleichberechtigung beider Sprachgruppen einzusetzen (Gstettner 2008: 47-50).

Die weiteren Versuche sind, Jahrzehnte später, auch der sozialdemokratischen Partei zuzuschreiben. Bekannt ist dabei die Rolle Hans Simas, der als Landeshauptmann Ende der 1960er-/Anfang der 1970erJahre versuchte Lösungen zu finden, die den Kärntner Slowen:innen ihre durch Artikel 7 des Staatsvertrags ${ }^{6}$ zugesicherten Rechte zweisprachiger topografischer Beschilderungen ermöglichten. Geendet hat der Versuch in seinem Rücktritt als Landeshauptmann und überhaupt aus der Politik, nachdem der Druck der Deutschnationalen - bekräftigt durch Attacken, Bombendrohungen und dem in die Geschichte eingegangenen Ortstafelsturm - zu groß wurde und seine Partei ihn nicht weiter unterstützte (Valentin 2013).

Als dritter Anlauf kann die aktuelle Zeit unter Landeshauptmann Peter Kaiser gezählt werden, der seit 2013 Landeshauptmann ist und beispielsweise in seinen

6 Der Artikel 7 des Staatsvertrags von 1955 regelt die "Rechte der slowenischen und kroatischen Minderheiten", also der Kärntner und Steirischen Slowen:innen sowie der Burgenlandkroat:innen in fünf Punkten: 1. „dieselben Rechte auf Grund gleicher Bedingungen [...] einschließlich des Rechtes auf ihre eigenen Organisationen, Versammlungen und Presse in ihrer eigenen Sprache“, 2. Schulwesen in den jeweiligen Sprachen, 3. Slowenisch und Kroatisch als Amtssprache und somit in den Verwaltungs- und Gerichtsbezirken dementsprechende topografische Bezeichnungen und Aufschriften, 4. Gleiche Bedingungen bei „kulturellen, Verwaltungs- und Gerichtseinrichtungen in diesen Gebieten", 5. Das Verbot der „Tätigkeit von Organisationen, die darauf abzielen, der kroatischen oder slowenischen Bevölkerung ihre Eigenschaft und ihre Rechte als Minderheit zu nehmen“ (Artikel 7, BGBl. Nr. 152/1955 idF BGBl. III Nr. 179/2002).
Reden immer wieder Worte auf Slowenisch spricht oder Veranstaltungen Kärntner-slowenischer Vereine besucht. Im Regierungsprogramm der Landesregierung 2013-2018, bestehend aus SPÖ, ÖVP und Grünen, wurde erstmals ein gleichberechtigtes Miteinander und ein Bekenntnis zu beiden Sprachen festgeschrieben. Darüber hinaus einigte sich dieselbe Landesregierung im Zuge einer Änderung der Landesverfassung auf folgenden Satz: „Die Fürsorge des Landes und der Gemeinden gilt den deutsch- und slowenischsprachigen Landsleuten gleichermaßen. " (Zitiert nach Rautz 2017: 157) Jedoch musste dieser Weg sogleich einen Rückschlag erfahren. Nach Veröffentlichung argumentierte die ÖVP, dass dieser Satz das Land spalte und die Bevölkerung diese symbolische Nennung - schließlich sind keine Rechte daran gebunden - angeblich nicht will. Daraufhin kam es zu weiteren Verhandlungen und einer Einigung, die erstmalig Deutsch als Landessprache festschreibt und Slowenisch als Landessprache dezidiert nicht nennt. Gerade weil die Formulierung in der Landesverfassung mehr symbolischen Wert als rechtliche Konsequenzen hat (Rautz 2017), ist diese Diskussion weder nachvollziehbar noch begrüßenswert.

Die Möglichkeit von Autonomierechten und Spracherhalt hängen mit dem Status der Sprecher:innen und der Sprache und damit verbunden ihrem möglichen politischen Rückhalt zusammen. Der zugesprochene Status hängt auch mit der sozialen Klasse zusammen.

\section{Minderheitenzugehörigkeit und Klasse}

Möglichkeiten, wie eine sprachliche Minderheit nachhaltig und langfristig bestehen kann, sind somit oft auch mit Fragen der Klassenzugehörigkeit verbunden. Unter sozialer Klasse wird in diesem Artikel die ungleiche Verteilung von Lebens-, Handlungs- und Teilhabechancen, die zumeist ökonomisch bestimmt ist, verstanden. Im Süden von Kärnten/Koroška (und der Steiermark/Štajerska) sprach die städtische, bürgerliche Schicht zumeist Deutsch und die breite, ländliche Bevölkerung Slowenisch. Zweitere waren häufig keine besitzenden Landwirt:innen, sondern zählten zum ärmeren Landproletariat, das lohnabhängig war. Ein Grund für diese demografische Entwicklung war, dass sich im mehrheitlich slowenischsprachigen Süden Kärntens/Koroškas das ,Freistiftrecht', ein zeitlich begrenztes Nutzungsrecht von Grund und Boden, länger hielt als das im vorwiegend deutschsprachigen Gebiet früher durchgesetzte ,erbliche Kaufrecht 
(Perchinig 1989: 17). Damit war es in den mehrheitlich slowenischsprachigen Gebieten lange schwieriger, zu Besitz zu kommen. „Sozialer Aufstieg war bereits zu Beginn des 19. Jahrhunderts mit Germanisierung verbunden, der Anteil der Slowenen an den höheren Schichten marginal." (ebd.: 19) Dazu kam in der zweiten Hälfte des 19. Jahrhunderts, neben dem Niedergang und Zusammenbruch der Eisenindustrie, eine (land-) wirtschaftliche Krise, welche vor allem (slowenischsprachige) Kleinbauern:Kleinbäuerinnen zum Verkauf des Besitzes zwang, welcher von (deutschen) Großgrundbesitzenden übernommen wurde (Larcher 1988: 15-17, 37-38, 53-55; Holzer 2008: 30-31).

Wie wichtig dieser Zusammenhang ist, zeigt sich auch in dem Umstand, dass bereits relativ früh repressiv gegen Teile der slowenischsprachigen Bildungselite vorgegangen wurde. Beispielsweise wurden während des Ersten Weltkriegs Priester und Lehrer:innen unter dem Vorwurf des Hochverrates verhaftet (Entner 2015: 10). Im Laufe der Zeit assimilierte sich vor allem das slowenischsprachige Landproletariat aufgrund des starken ökonomischen Drucks, während in landwirtschaftlich größeren Betrieben und damit wirtschaftlich selbstständigeren Familien die slowenische Sprache eher weitergegeben werden konnte (Neugebauer et al. 1990). Eine relative ökonomische Unabhängigkeit, wie es beispielsweise in der Landwirtschaft der Fall sein kann, fördert den Erhalt der Familiensprache, hier der slowenischen Sprache, während eine ökonomische Abhängigkeit Assimilation begünstigt (Gombos 1988: 129). Die sprachliche Assimilierung bietet zusätzlich, vor allem in sozial benachteiligten Verhältnissen, oft einen Schutz vor Diskriminierung (Jurić 1988: 185).

Interessant sind in dem Zusammenhang die Ergebnisse der letzten Volkszählung aus dem Jahr 2001, bei der auch die Umgangssprache erhoben wurde. ${ }^{7}$ Es konnte die eigene Umgangssprache freiwillig angegeben werden. Jene, die ,Slowenisch' angaben, haben im Alter ab 15 Jahren mit 10,6 Prozent eine deutlich höhere Akademiker:innenquote als jene, die ,Deutsch' angaben, mit 6,1 Prozent, oder jene, die ,Windisch' angaben, mit 1,5 Prozent. Bei Personen mit Pflichtschulabschluss als höchsten Bildungsabschluss drehen sich die Zahlen wieder um. So haben von jenen, die ,Windisch' angaben, 56 Prozent maximal Pflichtschulabschluss, von jenen, die ,Slowenisch' angaben, 39,3 Prozent, und von

7 Seitdem hat der Mikrozensus, eine Stichprobenerhebung, die die Umgangssprache nicht erfasst, die Volkszählung ersetzt. jenen, die ,Deutsch' angaben, 31,2 Prozent (Statistik Austria 2007). Es kann davon ausgegangen werden, dass die sich selbst als ,Windische“ Bezeichnenden zur eher assimilierten Gruppe gehören. Da der Bildungsabschluss oft ein Indikator für die soziale und ökonomische Stellung ist und Bildung vielfach ,vererbt ' wird, stützen die Ergebnisse der Volksabstimmung 2001 das Argument, dass Klassenfragen wichtige Faktoren zur Assimilation oder zum Erhalt der Sprache sind.

Die historisch gewachsenen ökonomischen Verhältnisse und damit die Wichtigkeit existenzsichernder Verbesserungen war einer der Hauptgründe, die zum Ausgang des Plebiszits für eine Republik und gegen eine Monarchie geführt haben. Schließlich versprach die Regierungsform einer Republik, im Gegensatz zu einer Monarchie, eine gewisse soziale Absicherung und mehr Gleichstellung.

\section{Versprechen von Kärntens Politik}

Die Sitzungsprotokolle der provisorischen Kärntner Landesversammlung und des Kärntner Landesrates zeigen, wie im Vorfeld des Plebiszits (10. Oktober 1920) regional sozialpolitische Besserstellungen diskutiert wurden. Die provisorische Kärntner Landesversammlung wurde auf Basis des Wahlergebnisses von 1911 zusammengesetzt. Nach dem Zusammenschluss der deutschnationalen Parteien zur Deutschdemokratischen Partei im November 1918 hatte diese eine relative Mehrheit neben den Christlichsozialen, der Sozialdemokratischen Arbeiterpartei und zwei Abgeordneten, die keiner Partei, sondern dem Militär angehörten. Kärntner Slowen:innen waren nicht vertreten.

Im Gegensatz zu der auf den alljährlichen 10.-Oktober-Feiern verwendeten Formulierung eines ,deutschen Sieges' ist einer der wichtigsten Aspekte für den Ausgang der Abstimmung, dass, wie bereits beschrieben, die slowenischsprachige Bevölkerung in den ländlichen Regionen gegenüber deutschen Großbauernhöfen oder der deutschsprachigen bürgerlichen Bevölkerung in den Städten häufig sozial benachteiligt war. Weitere ausschlaggebende Aspekte sind das Versprechen, die slowenische Sprache werde gleichberechtigt bestehen bleiben, sowie das bereits erwähnte drohende Abschneiden von den Städten Klagenfurt/ Celovec und Villach/Beljak, die (wirtschaftlich) für die Region wichtiger waren als beispielsweise die heutige Hauptstadt Sloweniens, Ljubljana.

Die Entscheidung für die Erste Republik Österreich ist an der Intersektion von Klasse und Spracherhalt 
anzusiedeln. Ein Vergleich der Protokolle aus der Zeit vor dem Plebiszit mit jenen nach der Abstimmung legt nahe, dass dies den in der Landesversammlung und im Landesrat vertretenen Parteien bewusst war. In Folge wird zuerst auf die Versprechen sozialer Rechte und dann auf jene gleicher (Sprach-)Rechte eingegangen. Dabei wird offensichtlich, dass viele jener symbolischen Versprechen, die der Abstimmung vorausgegangen waren, gleich danach nicht mehr von Bedeutung waren.

\subsection{Versprechen sozialer Rechte}

Die provisorische Landesversammlung debattierte im betrachteten Zeitraum einige Änderungen und Erweiterungen sozialer Rechte. So wurde Ende November 1919 rückwirkend ab 1. Jänner 1918 ein Gesetz zur Weitereinhebung der Wertzuwachsabgabe beschlossen, um Schulden $\mathrm{zu}$ tilgen und um in Schulen, Wohnungsfürsorge und Armenfonds investieren zu können (Landtag No XIII/124). Weitere sozialpolitische Gesetze, die auf Landesebene verabschiedet wurden, waren beispielsweise die Einrichtung eines Gesundheitsdienstes oder Lohnerhöhungen der Lehrenden an Volks- und Bürgerschulen (Landtag No XIII/129).

Es gab allerdings auch Anträge, die zur Verbesserung sozialer Rechte vor dem Plebiszit gestellt, aber nach dem 10. Oktober 1920 abgelehnt wurden. Als Beispiel sei hier die in der 56. Sitzung am 9. und 10. März 1920 (Landtag No XIII/136) eingebrachte „Schaffung eines Landesgesetzes für die Altersversorgung der landwirtschaftlichen Arbeiter und Dienstboten“ sowie die Einführung einer „obligatorischen Krankenversicherung der landwirtschaftlichen Arbeiter und Dienstboten“ im Rahmen der Kärntner Landesversicherung genannt. Beide Anträge wurden in der 69. Sitzung am 30. November 1920 als durch eine staatsgesetzliche Regelung überholt abgelehnt (Landtag No XIII/153). Ähnliches geschah mit dem Gesetzesentwurf für Beitragsleistungen der Heimatgemeinden zu den Verpflegungskosten für ihre in öffentlichen ,Kranken- und Irrenanstalten' versorgten zahlungsunfähigen Angehörigen, der in der 63. Sitzung am 11. Juni 1920 noch in den Finanzausschuss verwiesen wurde (Landtag No XIII/146), um in der 74. Sitzung vom 13. Dezember

8 Die Einhebung der Wertzuwachsabgabe wurde bereits 1911 eingeführt, damals aber noch mit der Ausnahme des Kaisers aus dieser Regelung (Landesgesetz- und Verordnungsblatt für das Herzogtum Kärnten, IX. Stück, 1911).
1920 als durch eine staatsgesetzliche Regelung überholt abgelehnt zu werden (Landtag No XIII/158).

Diese Beispiele können auch damit argumentiert werden, dass die Frage, was Landes- und was Bundeskompetenz ist, komplex ist, immer wieder diskutiert wird und besonders vor dem Inkrafttreten des BundesVerfassungsgesetzes am 10. November 1920 unklar war. Gerade in einer politischen Umbruchsphase kann daher die Unwissenheit über (Un-)Zuständigkeiten zu dementsprechenden Anträgen führen. Wird aber das jüngste Beispiel des Gemeinderates von Grafenstein/ Grabštanj betrachtet, der, wissentlich der Unzuständigkeit bei Änderungen von Bundesgesetzen, einen Ausstieg aus dem Minderheitenschulwesen beschlossen hat, ${ }^{9}$ ist klar, dass Anträge auch ohne faktische Kompetenz lediglich aufgrund der Symbolik eine Wirkkraft haben können. Vor allem hinsichtlich des Plebiszit 1920 ist im Wissen um die Effizienz von Symbolen nicht auszuschließen, dass die Anträge dementsprechend platziert wurden.

Eine besonders lang dauernde Diskussion gab es in dieser Zeit über die als veraltet und menschenunwürdig gesehene ,Dienstboten-Ordnung' von 1874 und einen damit einhergehenden Gesetzesvorschlag zur Erneuerung dieser. Damit sollte ein Mindestmaß an Arbeitsrechten für Land- und Forstarbeiter:innen, einer wichtigen Gruppe Abstimmungsberechtigter, sichergestellt werden, ohne darüber hinausgehende Regelungen auszuhebeln. Dafür wurde im Februar 1920 in der provisorischen Landesversammlung ein Antrag auf Verbesserungen der Arbeitsbedingungen von Landarbeiter:innen gestellt, mit Arbeitszeitregelungen (maximal zehn Stunden am Tag), regelmäßigem Lohn, Versicherungsleistungen, schriftlichem Vertrag, Einrechnung der Wegzeiten, Arbeitspausenregelungen, Sonntagsruhe, Feiertagsregelungen mit festgesetzten Feiertagen, Nachtarbeitsverbot, Überstundenregelungen, Mutterschutz und Kündigungsfristen (Landtag No XIII/131). Nach der Zuweisung zum Verfassungsausschuss im März wurde der Antrag im Mai dem Wirtschaftsausschuss zugewiesen (Landtag No XIII/140). In der 73. Sitzung vom 9. bis 11. Dezember 1920, also nach dem Plebiszit, kam es zu Parteienverhandlungen bezüglich der ,Landarbeiterordnung؛. Die Verhandlungen dauerten so lange, dass die Sitzung über mehrere Tage ging. Daraus resultierte ein Gesetz, das die Lage der Landarbeiter:innen zwar verbesserte, aber nicht

9 Siehe u.a. https://kaernten.orf.at/stories/3057071/ [13.9.2020]. 
annähernd in dem Umfang, wie der Antrag zu Beginn gestellt wurde. So wurden beispielsweise viele Ausnahmeregelungen zur Arbeitszeit eingeführt, damit die angedachten zehn Stunden immer wieder überschritten werden können. So wurde der „lichte Tag“ als Arbeitszeit festgesetzt, aber jahreszeitenspezifische Arbeiten, wie Spinnen, durften gegen Entschädigung von den Arbeiter:innen auch über den lichten Tag hinaus nicht abgelehnt werden. Des Weiteren wurden die Führung des Haushaltes oder mit Vieh verbundene Arbeiten aus allen Arbeitszeitregelungen ausgeschlossen - so auch aus der Sonn- und Feiertagsruhe. Feiertage wurden überhaupt keine mehr festgesetzt und der ursprüngliche Satz, dass Arbeiterinnen, wenn sie einen eigenen Haushalt zu führen haben, Zeit dafür brauchen und an Ostern, Pfingsten und Weihnachten frei bekommen, wurde so geändert, dass dies nur mehr für verheiratete Arbeiterinnen galt. Zudem wurde eine Mutterschutzregelung von zehn Wochen (acht davon nach der Geburt) auf 14 Tage nach der Geburt verkürzt (Landtag No XIII/157).

Neben den zielgruppenspezifischen Debatten bezogen auf das Landproletariat, das in der Zone A vielfach slowenischsprachig war, zeigt sich in den Protokollen, dass es der Landesversammlung wichtig war, positive Signale für Personen in und aus den Abstimmungszonen $\mathrm{zu}$ senden. So wurden Zuschüsse und Zuwendungen für Landes- und Staatsbedienstete in den betroffenen Gebieten und für Geflüchtete aus den Regionen beschlossen (Landtag No XIII/136; Landtag No XIII/140; Landtag No XIII/148). In den Protokollen des Landesausschusses findet sich ein Erlass des Staatsamtes für Finanzen an die Landesregierung, dass Steuerbegünstigungen für das durch die Abwehrkämpfe geschädigte Gebiet gewährt werden können. Dabei sollte bei den Bewohner:innen der Zone A von Österreich keine höhere Vermögensabgabe eingehoben werden als vom SHS. Der Landesrat beschloss zusätzlich, dass die vom SHS eingehobenen Steuern in die österreichische Vermögensabgabe eingerechnet werden. Dass der Ausgang der Abstimmung unklar war, zeigt sich daran, dass zeitgleich erlassen wurde, Investitionen nicht vor der Abstimmung zu beginnen (Kärntner Landesausschuss 4, 16. Sitzung, 22.4.1920). Selbige Begünstigungen wurden wenig später auch für die Zone B beantragt (Kärntner Landesausschuss 4, 22. Sitzung, 1.6.1920). Negative Signale wurden unterdessen vermieden. Beispielsweise wurde eine Anregung des Gemeinderates Klagenfurt/Celovec, den Mieter:innenschutz und die Arbeitslosenunterstützung für „Nicht-Deutschösterreicher“ aufzuheben mit der Begründung abgelehnt, Rücksicht auf die Lage der Stadt in der Abstimmungszone B zu nehmen, die Regelung allerdings einer zukünftigen Landesversammlung überlassen zu wollen (Landtag No XIII/142).

Die Achtsamkeit darauf, welche Signale im Vorhinein der Abstimmung vonseiten des Landes gesendet werden, lässt auf das Bewusstsein der Wichtigkeit wirtschaftlicher und sozialer Argumente schließen. Liest man Berichte slowenischer Intellektueller aus der Zeit nach dem Plebiszit, wird klar, dass die sozialpolitische Frage von jugoslawischer Seite massiv unterschätzt wurde und viel mehr auf die Frage einer nationalen Zugehörigkeit gesetzt wurde (Sammelarchiv Freiheitskämpfe 1918-1920 „Slow. Literatur“). Auch Nachrichten, wie mit demokratischen Rechten in der jungen SHS-Monarchie umgegangen wird, könnten diskursiv betrachtet mitausschlaggebend gewesen sein. Beispielsweise streikten im April 1920 in Slowenien Eisenbahner und aus Solidarität schlossen sich Bergarbeiter dem Streik an. Die Regierung des SHS antwortete mit einer militärischen Mobilisierung der Streikenden, um den Eisenbahnverkehr wiederherzustellen. Am 24. April 1920 eskalierte die Situation in Ljubljana, als die Polizei auf eine Demonstration schoss und 13 Menschen ums Leben kamen (ebd.). Die geografische und sprachliche Nähe Ljubljanas zum Abstimmungsgebiet, die zeitliche Nähe zum Plebiszit und die inhaltliche Nähe zu Fragen des politischen Systems in Verbindung mit den eigenen Rechten konnten Nachrichten wie diese zu meinungsbildenden Elementen machen.

\subsection{Versprechen gleicher Rechte}

Zusätzlich zu den sozialen Zusicherungen gab es im Vorfeld des Plebiszits Versprechen gleicher Rechte für Sprecher:innen beider Sprachen. So beschloss die vorläufige Kärntner Landesversammlung am 20. Juli 1920 einen Entschließungsantrag, in dem unter anderem steht: „Sie [die vorläufige Landesversammlung, Anm.] ist bereit die nationalen Wünsche der slovenischen Landesgenossen im Rahmen des durch den Friedensvertrag vorgesehenen Minderheitsschutzes zu erfüllen und ihnen den gewünschten Schutz zur Wahrung und Pflege ihrer Sprache und Nationalität im Sinne des Friedensvertrages angedeihen $\mathrm{zu}$ lassen." (Landtag No XIII/147) Um dies deutlich zu machen, beschloss der Landesrat am 3. September 1920, „eine Sitzung der Landesversammlung wenn nötig einzuberufen, um eine öffentliche Kundgebung wegen der Gewährung 
der Minderheitenrechte an die kärntnerische slavische Bevölkerung zu erlassen“ (Kärntner Landesausschuss 4, 33. Sitzung). Damit wird deutlich, dass man sich auch bei sprachlichen Rechten der Wirkung von Versprechen im Vorfeld des Plebiszits bewusst war.

Die angekündigte Sitzung fand drei Wochen später als 66. Sitzung der provisorischen Landesversammlung am 28. September 1920 statt. Weniger als zwei Wochen vor dem Plebiszit bekräftigte ein Entschließungsantrag die Gleichberechtigung der beiden Sprachgruppen. Konkret heißt es darin, dass die deutsche und slowenische Bevölkerung durch gemeinsame wirtschaftliche Interessen, Geschichte und Kultur eine Einheit seien und die provisorische Landesversammlung, im Gegensatz zu einer postulierten Willkür des SHS-Staates, eine „Politik der Versöhnung und Gerechtigkeit“ vertrete. „Sie erklärte daher im Bewusstsein der verantwortungsvollen Stunde namens der von ihr vertretenen Bevölkerung, dass sie den slovenischen Landsleuten ihre sprachliche und nationale Eigenart jetzt und allezeit wahren will, und dass sie derem geistigen und wirtschaftlichem Aufblühen dieselbe Fürsorge gedeihen lassen wird, wie den deutschen Bewohnern des Landes." (Landtag No XIII/149) Darüber hinaus wird festgehalten, dass die „Ausarbeitung dieser Grundsätze [...] nach durchgeführter Wiedervereinigung mit den Vertretern der Kärntner Slovenen vereinbart werden [wird]“, wofür auch die „demokratischen Grundsätze“ der Ersten Republik bürgen würden (ebd.). Interessant ist in diesem Entschließungsantrag der deutliche Hinweis auf die „Gemeinsamkeit wirtschaftlicher Interessen“, die „demokratischen Grundsätze“ der Republik, die der slowenischsprachigen Bevölkerung alle Rechte garantieren sollen, und darauf aufbauend einen versprochenen „wirtschaftlichen und kulturellen Aufschwung" ermöglichen. Dabei wird deutlich, wie eng die Argumente miteinander verwoben sind.

In der darauffolgenden 67. Sitzung nach dem Plebiszit, am 25. November 1920, wurde darauf eingegangen, dass sich die Abstimmung gegen eine Monarchie und für soziale Rechte gerichtet hatte. So erklärte der Vizepräsident der Landesversammlung, Julius Lukas, in seiner Eröffnungsrede, dass der Ausgang der Abstimmung eine „Ablehnung der Militärmonarchie SHS“ sei, und der Landesverweser ${ }^{10}$ Dr. Arthur Lemisch erläuterte in seiner Rede, dass „die Arbeiterschaft des Landes“, die „nicht Besitz und Eigentum zu verteidigen“ hatte, ausschlaggebend für den Ausgang

10 Heute der Landeshauptmann. aus Abwehrkampf und Volksabstimmung war (Landtag No XIII/150). Was vor wie nach der Abstimmung Bestand hatte, war das klare Bekenntnis gegen eine Monarchie. Selbst in der 86. Sitzung, am 6. April 1921, erklärte der Landesverweser im Namen aller Parteien aufgrund der Durchreise Karl Habsburgs durch Österreich nach Ungarn, dass Kärnten gegen alle Versuche der Rückkehr zu einer Monarchie sei und dass die „republikanische Gesinnung bei der Volksabstimmung deutlich zum Ausdrucke kam, treu und unerschütterlich zur österreichischen Republik steht und an der verfassungsmäßig festgelegten Staatsform festhält“ (Landtag No XIII/170).

Der argumentative Kern der inszenierten Festsitzung vom 25. November 1920 klang jedoch ganz anders als die im Vorfeld des Plebiszits gewählten Worte einer Gleichheit der Sprachgruppen. Die Reden sind dabei nicht nur in den Protokollen zu finden, sondern wurden am Tag darauf in der „Klagenfurter Zeitung“ ident abgedruckt. Sie deuten bereits an, was die Geschichte an gewaltvoller Germanisierung, vor allem während der Zeit des Nationalsozialismus, noch bringen wird. So findet sich in Lemisch' Rede ein deutlicher Deutschnationalismus, wie folgender Auszug illustrieren soll: „Bei der Wiederaufrichtung der Heimat dürfen nicht jene 15.278 vergessen bleiben, die beim Plebiszit für den Anschluß an SHS stimmten. Wir glauben, daß davon wohl viele Tausende Verführte sind, die wir wieder zu Kärntnern zu machen haben. [...] Nur ein Menschenalter haben wir Zeit, diese Verführten zum Kärntnertum zurückzuführen; in der Lebensdauer einer Generation muß das Erziehungswerk vollendet sein. [...] Die Kultur des deutschen Volkes hat Kärnten zur südlichen Mark gemacht, die Kultur Mitteleuropas gegenüber südlicher Hyperkultur soll es und wird es auch schaffen, mitzuhelfen, daß Kärnten ungeteilt bleibt. Mit deutscher Kultur und Kärntner Gemütlichkeit wollen wir, wenn Schule und Kirche das Ihre tun, in einem Menschenalter die uns vorgesteckte Arbeit geleistet haben." (Landtag No XIII/150) In den darauffolgenden Teilen der Rede argumentierte Lemisch widersprüchlich, indem er sagte, dass einerseits bereits 1848 klar war, dass Kärnten eine Nation mit zwei Sprachen sei und andererseits, dass das deutsche Volk das Beste und unzerstörbar sei (ebd.).

\subsection{Versprochen und gebrochen}

Die gemachten Versprechen der Gleichstellung wurden nicht nur verbal nach dem Plebiszit nicht eingehalten. 
Im Gegenteil - es wurden recht zeitnah slowenischsprachige Priester, Lehrer:innen, Beamt:innen, Angestellte des öffentlichen Dienstes und andere Personen, vor allem, wenn sie sich für die Stimmabgabe für den SHS-Staat eingesetzt hatten, entlassen, versetzt, vertrieben oder verhaftet. Das von Lemisch' kommunizierte „Umerziehungswerk" wurde sogleich gestartet. Jene slowenischsprechenden Personen, die in der jungen Republik blieben, wurden in „deutschfreundliche“ und „assimilierungswillige“ "Windische“ sowie "jugoslawisch orientierte“ und "heimatfeindliche“ Slowen:innen geteilt. Repression, Diskriminierung und Diffamierung zweiterer führten zu einem drastischen Rückgang jener, welche sich zur slowenischen Sprache bekannten (Scharsach 1992; Malle/Entner 2003; Rettl/ Blohberger 2014). Diese Zweiteilung wurde 1927 vom Historiker Martin Wutte in die "Windischentheorie“ verpackt, eine rassentheoretische Erklärung, die im Grunde besagt, dass ,Windische eigentlich (slowenischsprachige) Deutsche seien. Dies führte so weit, dass während des Nationalsozialismus 80 Prozent der Bevölkerung einer ganzen Gemeinde, St. Jakob im Rosental/Šentjakob v Rožu, vermessen wurde, um diese Theorie zu stützen (Koroschitz/Rož 2018).

Ihren traurigen Höhepunkt fand die Diskriminierung Kärntner Slowen:innen in den Deportationen, Verfolgungen und Ermordungen während des Nationalsozialismus. Doch auch in der Nachkriegszeit und in der Zweiten Republik war und ist die Frage der Gleichstellung eine stets umstrittene. Viele Rechte mussten juristisch und politisch erkämpft werden. Bis heute sind in Kärnten/Koroška weder Zwei- oder Mehrsprachigkeit eine Selbstverständlichkeit noch erfährt Slowenisch in der Öffentlichkeit eine gleichwertige Stellung wie Deutsch. Dies wurde im Sommer 2020 unter anderem wieder sichtbar, als die damalige Klagenfurter Bürgermeisterin Dr.in Maria-Luise Mathiaschitz die zweisprachige Beschriftung einer Sandkiste mit den Worten „Klagenfurt ist nicht zweisprachig!" kommentierte, sodass die Tafel umgehend abmontiert wurde. ${ }^{11}$ Dabei widerspricht diese Aussage zumindest der Nennung der Stadt Klagenfurt/Celovec im Minderheitenschulwesen-Ausführungsgesetz. Wenn bedacht wird, was nach dem Plebiszit an Dif-

11 Siehe u. a. https://www.kleinezeitung.at/kaernten/klagenfurt/5846988/Zum-Streit-um-eine-zweisprachige-Aufschrift_Wenn-sich-die-Politik oder https://www. kleinezeitung.at/kaernten/klagenfurt/584699o/Klagenfurt_Streit-um-zweisprachige-Aufschrift-auf-Sandkiste [16.9.2020] famierung, Diskriminierung, Hetze und Hass - bis zu Vernichtung(sgedanken) jenen, die sich als ,Slowenisch' bezeichneten, entgegenschlug, wundert es nicht, dass sich viele selbst lieber als ,Windisch bezeichnen, selbst wenn zeitgleich die Fremdbezeichnung als Beschimpfung empfunden wird. Allein die Möglichkeit der Repression kann im Hinblick auf Selbstschutz zur Assimilation führen (Clar 2016).

Sind die sozialpolitischen Versprechen der Zeit vor dem Plebiszit zum Teil umgesetzt worden, zum Teil in andere Kompetenzen gefallen und zum Teil nicht umgesetzt worden, so zeigte sich bei den Versprechen gleicher (Sprach-)Rechte, dass diese innerhalb kürzester Zeit nicht nur vergessen schienen, sondern sogar ins Gegenteil schlugen. Die Geschichte zeigt auf drastische Weise die Vulnerabilität von marginalisierten Gruppen.

\section{Mehrheitsmeinung und Minderheitenrechte}

Die Ausführungen wollen deutlich machen, dass Minderheitenpositionen von Mehrheitsmeinungen abhängig sind. Dabei geht es aber nicht unbedingt um quantitativ zählbare Größen. Unter Mehrheit wird, gemäß einer Hegemonie-theoretischen Interpretation, verstanden, dass deren Identität in eine Position gehoben wird, in der sie gewissermaßen ,das große Ganze repräsentiert (Laclau/Mouffe 1985; Laclau 1996, 2005; siehe auch Morozov 2020) bzw. ihr eigenes Interesse erfolgreich als das Interesse einer gesamten Gesellschaft etabliert (Gramsci 1971; siehe auch Morozov 2020). In Abgrenzung zur Mehrheit wird der Begriff ,Minderheit' als eine Positionierung verstanden, die es Individuen und Gruppen erschwert, hegemoniale Machtpositionen sowie einen gleichberechtigten Zugang zu Ressourcen, Möglichkeiten und vermeintlichen Selbstverständlichkeiten zu erlangen. Es geht in der Frage des Verhältnisses von Minderheit(en) und Mehrheit(en) also um die Frage von Macht, um den Zugang zu gesellschaftlichen Positionen, Möglichkeiten und Rechten.

Einer Assimilation der Minderheit, wie sie vielfach in Kärnten/Koroška geschehen ist, kann mit weitgreifenden Minderheitenrechten entgegengewirkt werden. Dazu benötigt es in einer Demokratie zumindest die Billigung dieser durch die Mehrheit, welche die hegemoniale Deutungsmacht über die Interessen einer Gesellschaft und damit auch über den Begriff, Minderheit' innehat. Diesen Begriff universell zu definieren, ist dementsprechend schwierig bzw. vom jeweiligen histo- 
rischen und politischen Kontext abhängig. Nicht einmal das „Rahmenübereinkommen zum Schutz nationaler Minderheiten“, welches in den späten 1990er-Jahren auf europäischer Ebene verhandelt und von vielen Staaten des Europarats ratifiziert wurde, kann eine klare Definition liefern, sondern überlässt dies, vor allem hinsichtlich der Frage, ob die Schutzbestimmungen lediglich autochthone oder auch neuere Minderheiten anspricht, den unterschiedlichen Vertragsstaaten (Pfeil 2016). In Österreich sind dies dieselben Gruppen wie jene, die in den Anwendungsbereich des Volksgruppengesetzes fallen, also die (burgenland-)kroatische, slowenische, ungarische, tschechische und slowakische ,Volksgruppe' sowie Rom:nja (BGBl. Nr. 38/1977; BGBl. Nr. 425/1992; BGBl. Nr. 895/1993).

Die Durchsetzung der Idee von Nationalstaaten macht Gruppen, die nicht in das hegemoniale Konzept der gezogenen Grenzen passen, zu Minderheiten. So geschehen auch in Österreich bei der Gründung der Ersten Republik infolge der Auflösung des Vielvölkerstaates der Habsburgermonarchie. Damit religiöse, sprachliche oder tradierte Unterscheidungen zur ,Mehrheitsbevölkerung' weiterhin gelebt werden können, benötigt es gewisse Rahmenbedingungen. Einen (juristischen) Schutz als Minderheit erfahren dabei aber oft nur jene, welche „autochthon (bzw. seit langer Zeit und geschlossen) auf einem bestimmten Territorium [leben]“ (Žagar 2011: 195). Die Differenzierung in , autochthon' und ,allochthon', in ,angestammte und daher mehr zu akzeptierende sowie, zugewanderte und daher weniger förderungswürdige Minderheiten stellt allerdings ebenfalls eine Hierarchisierung dar (Wieser 2014: 95). Menschen, die an einem Ort leben und diesen gestalten, haben unterschiedliche Zugänge zu Ressourcen. Dabei kann sich die Positionierung als Minderheit oder Mehrheit je nach Kontext dynamisch gestalten, was heißt, dass beispielsweise ,neue Minderheiten ' oftmals eine noch viel marginalisiertere Stellung zugewiesen bekommen. „[S]trukturelle Machtverhältnisse [müssen] als solche benannt werden, damit auch strukturelle Diskriminierungen nicht aus dem Blick geraten" (ebd.: 182)

Dem Rahmenübereinkommen und dem Volksgruppengesetz ist gleich, dass sie die Bekenntnisfreiheit betonen und jede Person somit selbst entscheiden kann, ob sie in den gesetzlichen Schutzbereich fallen möchte oder nicht (\$1 Absatz 3 BGBl. Nr. 396/1976; Artikel 3 Absatz 1 BGBl. III Nr. 120/1998). Die Geschichte der Kärntner (und Steirischen) Slowen:innen zeigt, wie oben über die Zweiteilung Windisch-Slowenisch dar- gestellt, dass ein Bekenntnis zu Repressionen führen kann und daher die freie Entscheidung, gerade im Hinblick auf Selbstschutz, wichtig ist. Dieser Passus widerspricht allerdings der Praxis, in der Gesetzgeber:innen (und Mehrheiten) oft quantitative Erhebungen als Grundlage für die Gewährung bestimmter Rechte voraussetzen. Damit drängt die Mehrheit Angehörige der Minderheit zum geforderten Bekenntnis. Die Verknüpfung von Zahlen an Rechte ist auch Teil der Entstehung des Volksgruppengesetzes. So wurde 1976, gegen den Willen von Vertreter:innen autochthoner Minderheiten, das Volksgruppengesetz beschlossen, welches Rechte an eine hohe Prozentzahl sich bekennender Sprecher:innen bindet. Um das Gesetz umsetzen zu können, wurde eine sogenannte Minderheitenfeststellung durchgeführt (Österreichisches Volksgruppenzentrum 2001: 18-20; Obid et al. 2002: 140). Diese wurde sowohl von Angehörigen der Minderheiten als auch von solidarischen Personen im Rest Österreichs nach dem Aufruf von slowenischen Vertretungsorganisationen und Verbänden boykottiert und ad absurdum geführt $^{12}$ (Perchinig 1989: 141-142; Mittersteiner 2010: 24; Valentin 2013: 510). Trotzdem wurden die errechneten Prozentzahlen in weiterer Folge für die Billigung von Rechten herangezogen (Obid et al. 2002: 45). Darüber hinaus mussten viele Rechte trotz gesetzlicher Schutzbestimmungen ${ }^{13}$ auf eigene Initiative gerichtlich durchgesetzt werden. ${ }^{14}$

12 Bspw. wurden bei dieser Zählung in Wien mehr Personen mit slowenischer Umgangssprache gezählt als in Kärnten/Koroška.

13 Bspw. Artikel 7-8 Bundes-Verfassungsgesetz, Artikel 19 Staatsgrundgesetz 1867, Artikel 66-68 Vertrag von Saint-Germain 1919, Artikel 7 Staatsvertrag 1955, Minderheiten-Schulgesetz für Kärnten 1959, Volksgruppengesetz 1976, Europäische Charta der Regional- und Minderheitensprachen 1992, Europäische Rahmenkonvention zum Schutz nationaler Minderheiten 1995 und 1998 unterzeichnet, Staatszielbestimmung zum Schutz der Volksgruppen 2000/2001.

14 Wie eine öffentliche zweisprachige Volksschule in Klagenfurt/Celovec (1989/1991), möglicher zweisprachiger Unterricht auch in der vierten Klasse Volksschule (2000), die Amtssprachenverordnung (2000/2001) sowie die Aufstellung topografischer Aufschriften wie Ortstafeln (Obid et al. 2002: 39). Bei Letzterem stellt sich allerdings die Frage, was als topografische Aufschrift gilt, denn Beschriftungen wie ,Gemeindeamt', ,Grenzübergang' oder von Straßenbeschilderungen sind ebenso zumeist einsprachig wie „Ortsbezeichnungen in Telefonbüchern, Fahrplänen, Kartenwerken u.ä. ,halbamtlichen` Druckwerken“ (Österreichisches Volksgruppenzentrum 2001: 38). 
Das Zählen von Minderheiten in einem nationalstaatlichen Rahmen geht immer mit machtpolitischen Fragen einher. Diese beziehen sich auf eine „Messung des Grades der Assimilation [mit] der Ausrichtung gezielter Assimilationsmaßnahmen, sowohl mit positiven wie negativen Sanktionen“ (Boysen 2011: 8) und gehen bis zu einem Interesse an „der physischen Vernichtung“ (ebd.). Die Existenz von Minderheiten bedroht die Fiktion „der ethnischen Homogenität des jeweiligen Staates“ (Žagar 2011: 174).

Die beschriebenen Machtungleichheiten führen zu einem Spannungsverhältnis in demokratischen Gesellschaften. Der oben beschriebene Passus der eigenständigen und freiwilligen Entscheidung, sich selbst als Teil einer Minderheit zu definieren, um Zugeständnisse aus der Mehrheitsgesellschaft zu bekommen, zeugt in ambivalenter Weise auch von der Abhängigkeit, sich überhaupt bekennen zu müssen - Menschen aus der hegemonialen Gruppe haben das Privileg, sich nicht als zugehörig offenbaren zu müssen. Das heißt, bei Angehörigen von Minderheiten „bedarf [es] des kollektiven Willens zum Erhalt der eigenen, spezifischen Identität" (Pfeil 2016: 625). Die Herausforderung dieser Abhängigkeit ist Teil des in einer Demokratie vorherrschenden Mehrheitsprinzips. Eine gleichberechtigte Partizipation, die von Mehrheitsverhältnissen abhängig ist, kann einer meist zahlenmäßig unterlegenen Gruppe nur dann garantiert werden, wenn es eigene Rechte und Möglichkeiten der Organisierung gibt. Dabei gibt es international gesehen unterschiedliche Beispiele, was das alles (für autochthone Minderheiten) beinhalten kann, wie „zB Ausnahmen von Prozentklauseln im Wahlrecht, garantierte Sitze für Minderheitenparteien oder -vertreter, Vorkehrungen der Territorial- oder Personalautonomie oder föderale Strukturen im Einklang mit Minderheiten-Siedlungsgebieten" (ebd.: 630).

Föderale Strukturen sind bekannt als Beispiele der Selbstverwaltung, was bedeutet, dass Teile der Gesetzgebung und Verwaltung, seltener der Gerichtsbarkeit, zumeist aber der Sprachpflege und Bildung in einem demokratisch legitimierten Aufgabenbereich innerhalb der Minderheit angesiedelt werden. Dabei ist diese Autonomie trotz allem an die Verfassung und Rechtsstaatlichkeit des jeweiligen Staatsgebietes gebunden. Antidiskriminierung und Gleichbehandlung sind dabei das Mindestmaß an Minderheitenrechten, echte Partizipation oder überhaupt Autonomie inklusive der Übertragung von Hoheitsrechten bedeuten darüber hinausgehende Rechte (Küpper 2013). Bekannte Bei- spiele für Autonomielösungen in Europa sind Südtirol oder die deutschsprachige Gemeinschaft im Osten Belgiens.

Doch auch ohne Einführung einer Autonomie kann in gegebenen Strukturen die Repräsentation von Minderheiten berücksichtigt werden. So ist beispielsweise Kärnten/Koroška in vier Wahlkreise eingeteilt, wobei in jedem Kärntner Slowen:innen leben. Bis 2008 war das Erreichen eines Grundmandates Voraussetzung für den Einzug in den Landtag, was faktisch zu einer umgerechneten Sperrklausel zwischen 9,09 und 11,11 Prozent der Stimmen im jeweiligen Wahlkreis führte (Lantschner 2009). Allerdings war es aufgrund der Aufteilung für slowenische Parteien unwahrscheinlich, ein Grundmandat zu erreichen. Die Sperrklausel, eine festgesetzte Marke, ab welcher Prozentzahl Parteien oder Listen in die jeweiligen Gremien einziehen können, hat einen Einfluss darauf, wie minderheitenoder mehrheitenfreundlich das Wahlsystem ist. In Kärnten/Koroška liegt diese seit 2008 bei 5 Prozent. In Schleswig-Holstein ist beispielsweise die Sperrklausel für die Partei der dänischen Minderheit ausgesetzt, solange sie zumindest die Stimmzahl für das Erreichen eines Mandates erhält.

Ein qualitatives Demokratieprinzip geht davon aus, dass Stimmen unabhängig von ihrer Quantität Eingang in parlamentarische Prozesse finden können. So haben die ungarische und die italienische Minderheit im slowenischen Parlament je ein zusätzliches, fix von ihnen zu wählendes Mandat. Es gäbe aber auch die Möglichkeit einer ,Konkordanzdemokratie', einem Modell, das darauf abzielt, möglichst viele Personengruppen einzubeziehen, ohne sich gegenseitig zu überstimmen. Dabei braucht es nicht unbedingt ein Mandat, sondern dies könnte beispielsweise in Form einer im Parlament eingerichteten Lobbyposition, deren primäre Aufgabe es ist, Themen, die die vertretene Minderheit betreffen, einzubringen und regelmäßig an deren Interessen $\mathrm{zu}$ erinnern, verwirklicht werden (Tichy 2002). In Österreich könnte das durch eine Stärkung der Volksgruppenbeiräte erreicht werden.

\section{Fazit}

Das Ziel dieser Auseinandersetzung war, die Intersektion von Klasse, Status und Möglichkeiten des Erhalts von Sprache sowie der Selbst- und Mitbestimmung aufzuzeigen. Dabei wurde ersichtlich, dass existenzsichernde Rechte und die Ausgestaltung von weitgreifenden Minderheitenrechten wichtig sind, um eine
IIIOMENTUM QUARTERLY (IIIIIIIIIIIIIIIIII

45 
gleichberechtigte Partizipation $\mathrm{zu}$ ermöglichen. Es wurde deutlich, dass eine doppelt marginalisierte Position Anpassungsstrategien, wie Assimilation, fördert. Die Betrachtung bestätigt somit, was Intersektionalitätsdebatten, und innerhalb jener vor allem schwarze Frauen und women of colour, seit Jahrzehnten fordern, nämlich, dass es notwendig ist, Schnittstellen unterschiedlicher Diversitätsdimensionen gemeinsam und herrschaftskritisch zu analysieren.

Die Staatsform der Ersten Republik in Österreich war bis 1934 eine Demokratie. Wenn unter Demokratie die alleinige Herrschaft der Mehrheit verstanden wird, kann das erhebliche Nachteile in Bezug auf Minderheitenrechte haben. Weniger Mitbestimmung führt zu weniger Möglichkeiten, Rechte, Verantwortung und Gestaltungsmacht $\mathrm{zu}$ erlangen und $\mathrm{zu}$ übernehmen. Dies wiederum kann einen negativen Einfluss auf Status und Wichtigkeit haben, was abermals dazu führt, dass tendenziell weniger Mitbestimmung ermöglicht wird. Wenn ,Minderheitenangehörige ' sozial benachteiligt sind, verschärft sich das eben Beschriebene. Zugeständnisse von Rechten an Zahlen zu knüpfen, während Bekenntnisse in ökonomischen Abhängigkeitsverhältnissen fragil sind, kann daher keine Lösung sein.

Anhand des 100 Jahre alten Beispiels des Plebiszits in Kärnten/Koroška lässt sich zeigen, wie relevant sozialpolitische Fragestellungen, vor allem sozial- und arbeitsrechtliche Aspekte, beispielsweise in der Wahl eines politischen Systems, sind. Die existenzielle Grundlage ist wortwörtlich eine überlebenswichtige Entscheidungsgrundlage. Gemeinsam mit dem Versprechen gleicher Rechte sind soziale Rechte und wirtschaftliche Anbindung ein Kernstück der damaligen Entscheidung der Bevölkerung in der Abstimmungszone A für die Republik Österreich. Doch wurden weder alle sozialen Rechte (zeitnah) umgesetzt noch die Versprechen der Gleichberechtigung gehalten. Demokratische Grundsätze alleine sagen noch nichts über deren Ausgestaltung aus.

Die folkloristische Ausrichtung der jährlich wiederkehrenden 10.-Oktober-Feiern haben von Anfang an den sozial- und wirtschaftspolitischen Aspekt ausgelassen, lenkt er doch ab von tradierten ,nationalen Fragen' und ,deutschen Siegen. Es war keine Abstimmung einer gegen die andere Sprachgruppe. Viel pragmatischer ging es um Fragen, wo Existenzen besser gesichert werden können, wo es einfacher ist, eine Arbeit zu finden oder zu halten, und wo es mehr Möglichkeiten an Rechten und Mitbestimmung auch für die sozial benachteiligte Bevölkerung gibt. Es hätte der Republik nicht geschadet, wenn die Versprechen gehalten worden wären.

\section{Literatur}

Boysen, T. (2011): Die Vermessung von Volksgruppen: Menetekel oder positive Diskriminierung? Nationale Minderheiten in Deutschland zwischen Bekenntnisprinzip und Social Engineering. In: Karpf, P./Kassl, T./Platzer, W./Puschnig, U. (Hg.): Zählen Minderheiten? - Volksgruppen zählen! Celovec/Klagenfurt: Amt der Kärntner Landesregierung, Volksgruppenbüro/Biro za slovensko narodno skupnost, 7-17.

Bourdieu, P. (2005): Was heißt Sprechen? Zur Ökonomie des sprachlichen Tausches. Mit einer Einführung von John B. Thompson (2. erweiterte und überarbeitete Auflage). Wien: Braumüller

Busch, B. (2008): Mehrsprachige Bildung in Österreich: Ein Fokus auf Curricula, Lehr- und Lernmaterialien. Online: https://heteroglossia.net/fileadmin/user_upload/publication/buscho8_mehrspra_schuleA.pdf [13.09.2020].

Clar, M. (2016): Zur Nicht-Weitergabe der slowenischen Sprache in Koroška/Kärnten. Eine biografieanalytische Annäherung. Wien: Masterarbeit Universität Wien.

Entner, B. (2015): Kärntner Slowenen und Sloweninnen - unbekannte/ungeliebte Minderheit im Süden Österreichs. Psychologie und Gesellschaftskritik, 39 (4), 7-31.

Gombos, G. (1988): Sprache zwischen Schicksal und Wahl. Umgangsformen mit der Sprache der Kindheit. In: Boeckmann, K.-B./Brunner, K.-M./Egger, M./Gombos, G./Jurić, M./Larcher, D. (Hg.): Zweisprachigkeit und Identität. Celovec/Klagenfurt: Drava, 125-152.

Gramsci, A. (1971): Selections from Prison Notebooks. London: Lawrence \& Wishart.

Gstettner, P. (2008): Ein deutscher Sieg und seine Folgen. Deutungen, Umdeutungen und Zwiespältigkeiten beim Erzählen der Kärntner Geschichte. In: Malina, P./Renner, E. (Hg.): Blickwechsel oder: Eine andere Erste Republik? Innsbruck/Wien/Bozen: Studienverlag, 56-76.

Holzer, A. (2008): Der Orttafelstreit in Kärnten in Hinblick auf das Wahljahr 2006. Ursachen, verdeckte Konflikte, Instrumentalisierung und Bedeutung in der Gegenwart. Wien: Diplomarbeit Universität Wien.

Jurić, M. (1988): Der Mann als ich, die Frau als wir. Unterschiedliche Weisen, ethnische Identität auszudrücken. In: Boeckmann, K.-B./Brunner, K.-M./Egger, M./Gombos, G./Jurić, M./Larcher, D. (Hg.): Zweisprachigkeit und Identität. Celovec/Klagenfurt: Drava, 153-188.

Kärntner Landesausschuss 4, AT-KLA 409-4 Su: Sitzungsprotokolle des Kärntner Landesrates.

Koroschitz, W./Slowenischer Kulturverein SPD Rož (2018): Vermessung / Meritev. Über die „rassenkundliche“ Untersuchung in St. Jakob im Rosental / O, ,rasoslovni“ preiskavi v Šentjakobu v Rožu. Celovec/Klagenfurt: Mohorjeva/ Hermagoras. 
Laclau, E. (1996): Emancipation(s). London: Verso.

Laclau, E. (2005): On Populist Reason. London: Verso.

Laclau, E./Mouffe, C. (1985): Hegemony and Socialist Strategy. London: Verso.

Landtag No XIII, Kärntner Landesarchiv, AT-KLA 145-C2.3.10 Ak: 12: Niederschriften der 41.-92: Sitzung (Abschriften).

Lantschner, E. (2009): Wahlgesetzgebung und ihre Auswirkung auf die Vertretung von Minderheiten: Die Kärntner Landtagswahlordnung im europäischen Vergleich. European Diversity and Autonomy Papers - EDAP o1.

Larcher, D. (1988): Soziogenese der Urangst. In: Boeckmann, K.-B./Brunner, K.-M./Egger, M./Gombos, G./Jurić, M./ Larcher, D. (Hg.): Zweisprachigkeit und Identität. Celovec/Klagenfurt: Drava, 15-64.

Malina, P./Renner, E. (2008): Die Erste Republik. Ein Annäherungsversuch in 12 Stationen. In: Malina, P./Renner, E. (Hg.): Blickwechsel oder: Eine andere Erste Republik? Innsbruck/Wien/Bozen: Studienverlag.

Malle, A./Entner, B. (2003): Anmerkungen zur Broschüre „Die Kärntner Slowenen", hg. vom Amt der Kärntner Landesregierung, Volksgruppenbüro/Biro za slovensko narodno skupnost. Online: http://docplayer.org/29754392-Anmerkungen-zur-broschuere-die-kaerntner-slowenen -hg-vom-amt-der-kaerntner-landesregierung-volksgruppenbuero-biro-za-slovensko-narodno-skupnost. html [14.11.2020].

Mittersteiner, M. (2010): Konflikt-Dialogbereitschaft-Stagnation. Schlüsselkomponenten des aktuellen Diskurses zur slowenischsprachigen Minderheit Kärntens. Wien: Diplomarbeit Universität Wien.

Morozov, V. (2020): New Nationalisms and Identity Politics: Minorities, Majorities and Universal Emancipation. European Review. DOI:10.1017/S1062798720000484.

Neugebauer, W./Messner, M./Pittler, A./Verdel, H. (1990): Spurensuche. Erzählte Geschichte der Kärntner Slowenen. Wien: ÖBV.

Obid, V./Messner, M./Leben, A. (2002): Haiders Exerzierfeld. Kärntens SlowenInnen in der deutschen Volksgemeinschaft. Wien: Promedia.

Ogris, T. (2010): Kärnten. Zwei Sprachen, eine Kultur. Koroška. Dva jezika, ena kultura. In: Amt der Kärntner Landesregierung, Volksgruppenbüro/Biro za slovensko narodno skupnost (Hg.): Gemeinsam 20 Jahre. Skupno 20 let. Volksgruppenbüro. Biro za slovensko narodno skupnost. Celovec/Klagenfurt, 26-134.

Österreichisches Volksgruppenzentrum. (2001): I am from Austria - Volksgruppen in Österreich. Wien: Mohorjeva/ Hermagoras.

Perchinig, B. (1989): Wir sind Kärntner und damit hat sich's... Deutschnationalismus und politische Kultur in Kärnten. Celovec/Klagenfurt: Drava.

Pfeil, B. S. (2016): Was ist eine „Minderheit“? Von „,alten Minderheiten“, „neuen Minderheiten“ und Sinn und Grenzen einer völkerrechtlichen Minderheitendefinition. Europäisches Journal für Minderheitenfragen, 3-4, 614-637.
Rautz, G. (2017): Die neue Kärntner Landesverfassung - Eine Posse in drei Akten. Europäisches Journal für Minderheitenfragen, 1-2, 156-172.

Rettl, L./Blohberger, G. (Hg.) (2014): Peršman. Göttingen: Wallstein.

Sammelarchiv Freiheitskämpfe 1918-1920 „Slow. Literatur“, Kärntner Landesarchiv, AT-KLA 908.

Scharsach, H.-H. (1992): Haiders Kampf. München: Wilhelm Heyne.

Statistik Austria (2007): VOLKSZÄHLUNG 2001. Textband. Die demographische, soziale und wirtschaftliche Struktur der österreichischen Bevölkerung. Wien.

Tichy, H. (2002): Die parlamentarische Vertretung von ethnischen Minderheiten und das österreichische Verfassungsrecht. Parliaments, Estates \& Representation, 22 (1), 201-213.

Valentin, H. (2013): Am Rande des Bürgerkrieges. Der Kärntner Ortstafelkonflikt 1972 und der Sturz Hans Simas. Celovec/ Klagenfurt/Ljubljana/Wien: Mohorjeva/Hermagoras.

Wieser, V. (2014): Ambivalenzen der Gemeinschaft. Normen, Abgrenzungen und Ausschlüsse in der kollektiven Identitätsbildung der Kärntner Slowen:innen. Eine theoretische Abhandlung mit empirischen Beispielen aus der kärnterslowenischen Wochenzeitung „Novice“. Wien: Masterarbeit Universität Wien.

Wohlfahrter, E. (2014): „Stadtluft macht frei, auch sprachlich“: Sprachbiographische Gespräche mit Kärntner SlowenInnen in Wien. Wien: Masterarbeit Universität Wien.

Žagar, M. (2011): Nationale Minderheiten, Minderheitenschutz und Partizipation: Entwicklungsperspektiven in den Nachfolgestaaten des ehemaligen Jugoslawien. In: Karpf, P./Kassl, T./Platzer, W./Puschnig, U. (Hg.): Zählen Minderheiten? - Volksgruppen zählen! Celovec/Klagenfurt: Amt der Kärntner Landesregierung, Volksgruppenbüro/Biro za slovensko narodno skupnost, 171-288. 